\title{
FLUIDIC GENERATOR OF MICROBUBBLES - OSCILLATOR WITH GAS FLOW REVERSAL FOR A PART OF PERIOD
}

\author{
Václav TESA $\check{R}^{*}$ \\ "Institute of Thermomechanics v.v.i., Academy of Sciences of the Czech Republic, Dolejškova 1402/5, 18200 Praha 8, Czech Republic \\ tesar@it.cas.cz
}

received 10 June 2015, revised 11 December 2015, accepted 14 December 2015

\begin{abstract}
Paper presents a fluidic device developed for generation of small (less than $1 \mathrm{~mm}$ in diameter) microbubbles in a liquid from gas passing gas through small passages. Until now the bubbles are larger than the size of aerator passage exits so that making the passages smaller did not result in obtaining the desirable microbubbles. Analysis of high-speed camera images (obtained with a special lens of large working distance) have shown show that the large bubble size is caused by slow ascent motion of very small bubbles so that they get into mutual contact and grow by conjunction. The solution is to pulsate the supplied gas flow by a no-moving-part fluidic oscillator. The generated small bubble is moved back into the aerator passage where it is for a part of oscillation period protected from the conjunction with other, previously generated microbubbles.
\end{abstract}

Keywords: Fluidics, Oscillators, Microbubbles, U-Tube Resonator

\section{INTRODUCTION}

An important development has been recently taking place in areas of engineering working with gas bubbles in liquids. The key factor is introduction of energetically efficient generation of microbubbles, of diameter less than $1 \mathrm{~mm}$ (Rodríguez-Rodríguez et al., 2015) - but larger than $1 \mu \mathrm{m}$ so that they should not be confused with differently behaving smaller nanobubbles (Prevenslik 2011, Zimmerman et al., 2011). Microbubbles can intensify and accelerate several processes.

\subsection{Applications and importance of microbubbles}

An area in which microbubbles have already demonstrated their intensification capability are processes that depend on diffusion transport of gas into liquid. The large total surface of microbubbles, together with longer diffusion time (due to low microbubble ascent velocity), lead to unprecedented high transfer rates. A typical example of the intensification is the increased transfer of oxygen into the liquid in waste water processing (Terasaka et al., 2011, Rehman et al., 2015, Rawat et al., 2011). The decomposition of organic waste is actually done by aerobic bacteria, the metabolism of which is roughly $90 \%$ more intensive than that of anaerobic ones. Aeration to provide the aerobic bacteria with the oxygen they need is the most energy consuming activity in waste water treatment plants. The improvement achieved by microbubbles enhances substantially the overall efficiency of the treatment process.

An increase in effectiveness was also demonstrated in the use of microbubbles for separation of substances by flotation (Coward et al., 2015, Hanotu et al., 2012, 2013). Microbubbles, by their strong clinging to contaminants like oil or grease, make possible efficient and environmentally friendly removal of fats in product cleaning. Microbubbles also exhibit a remarkable washing effect without chemical detergents. For example, Watanabe et al., (2013) describe use of microbubbles for de-contamination of silicon wafers. Microbubbles were also demonstrated by $\mathrm{Xi}_{\mathrm{i}}$ (2012), to be efficient for disinfection by removal of pathogens. Coward et al., (2015) demonstrated efficient flotation harvesting of algae grown in bioreactors for producing biofuels.

In food industry microbubbles can extend shelf life of products of foamy character - whipped cream, ice-cream, sorbets, and mousses - in addition giving them interesting taste properties. Microbubble foam was demonstrated to remain stable for up to a year. Also of interest for food industry is water evaporation ability of microbubbles (Zimmerman et al., 2013) without the heat reaching the dried product.

Hydrodynamic resistance of ships may be reduced by air microbubbles, as demonstrated by McCormick and Bhattacharyya already in 1973 having shown a 30\% reduction in friction drag. Their lack of further progress was due to inefficient generation of microbubbles -- by electrolysis, needing more energy than was saved in vessel propulsion. This problem of energetically efficient microbubble generation plagued also other similar attempts, including those able to show reductions by up to $80 \%$ (Bogdevich et al., 1978; Madavan et al., 1984). Recent tests with microbubbles were made with very large models (Watanabe et al., 1998; Moriguchi and Kato, 2002; Yanuar et al., 2012), at Reynolds numbers near to those of practical use. Of interest to process engineers is analogous decrease of friction in tube flows (Pang et al., 2014, Shams et al., 2014)

A wide and increasing spectrum of microbubble uses is currently in medicine. Microbubbles can convert energy of ultrasonic vibration into a local thermal therapeutic effect (Kanagawa, 2013). Streaming effect on microbubbles in ultrasonic field can destroy 
cells (Kooiman et al., 2011) and can cause their outer membranes to become permeable for drugs (Oh et al., 2014) particularly anticancer ones (Watanabe et al., 2008). Microbubbles are also used in measuring absolute blood pressure (Tremblay-Darveau et al., 2014).

Many currently developed uses of microbubbles aim at use in microfluidics. They are used in gene manipulation (Sun et al., 2014), biosensors (Kuznetsova and Coakley, 2007), mixing of reactants for chemical reaction (Lee et al., 2012) or sorting (Wang, 2012). Important progress is also being done in optofluidics, where ordered arrays of microbubbles can create tuneable optical components (Hashimoto et al., 2006; Allouch et al., 2014).

The most important current problem of geopolitics is the distribution of cheap crude oil sources in individual parts of the world. The sources are in politically unstable regions, e.g., in Near East, but absent in the areas of most consumption (Europe, North America). A solution is envisaged in producing liquid fuels from lipids in algae (Lam and Lee, 2012; Leite et al., 2013; Kargbo 2010). The processes used actually simulate the way by which fossil crude oil was produced 300 million years ago. Decisive factor for success of this idea is increasing the economy of all algae growing and processing steps. One of these steps, seemingly secondary but in fact quite important, is supplying $\mathrm{CO} 2$ which algae need for photosynthesis. In photobioreactors. This is done by bubbling the gas through the algae-containing liquid column (Hu et al., 2015), where an improvement of the diffusion transport by $\mathrm{CO}_{2}$ microbubbles may be an essential enhancement. Another perhaps less obvious effect achieved by the gas bubbles in bioreactors is removal of $\mathrm{O}_{2}$ produced by the algae. Oxygen is an inhibitor to microalgae growth and at saturation level it is toxic. Zimmerman et al. (2011) demonstrated that $\mathrm{O}_{2}$ removal leads to much higher algae growth rates.

\subsection{New microbubble generation method}

In view of the above list of promising microbubble uses (by far not complete), there is no wonder interest in them has been propagating rapidly. Many advantages were actually obvious already earlier, but there was the hindrance of low microbubble generation cos. Considering the water containing environment it has been also desirable to avoid in the microbubble generator any operations by electric current.

The simplest and seemingly fail safe generation method is percolating the gas through an aerator - a body having a large number of parallel exit channels of very small cross section areas. Experience with this approach, however, were generally a failure. As demonstrated in the example Fig. 1, the bubbles obviously tend to grow far beyond the size of aerator exits.

A promising new solution was recently found (Tesař, 2007; Zimmerman et al., 2008) in pulsating the gas flow supplied into the aerator. Especially attractive is using for this purpose a nomoving-part fluidic oscillator - robust, inexpensive, with long life, high reliability, no maintenance demands, and operation without driving electric current. The oscillator is practically nothing more than just a special shaping of the gas (air) aerator entrance. Practical results obtained were, however, rather uncertain. Some experiments have demonstrated successful microbubble production - while in other attempts this approach failed. The main obstacle in the way towards success has been the lack of understanding the bubble generation mechanism and the way it is influenced by the fluidic oscillation. Initially, attempts focused on fragmentation effect, which is known to generate in the analogous (but, of course, inverse) problem of small liquid droplets in air (James et al., 2003; Tesař, 2014). Efficient action of the oscillation on a fragmented bubble requires applying it at natural resonant frequencies of the resultant microbubbles. These frequencies are, however, very high - in the kilohertz range, which obviously are not the frequencies at which the fluidic oscillators in the performed tests operated — which were typically $100 \mathrm{~Hz}$ to $200 \mathrm{~Hz}$. Higher frequencies are, in principle achievable, but designing fluidic oscillators for the kilohertz range (at reasonably large size) is far from easy. Evidently, the successful cases excited (Tesař, 2013b) higher harmonics of the basic frequency.

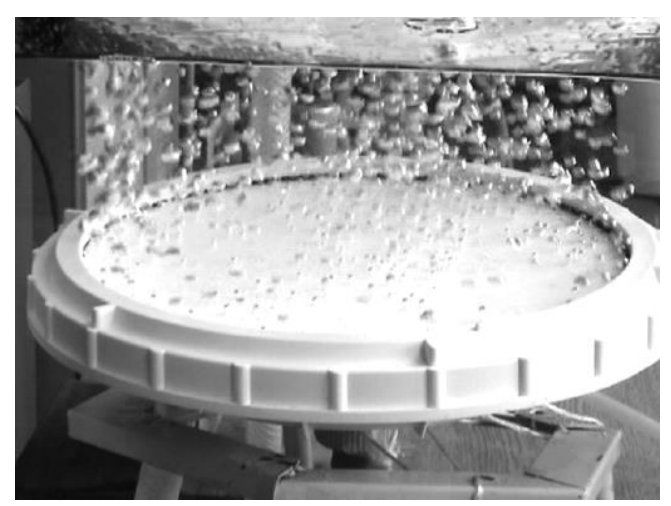

Fig. 1. An example of a typical present-day no-fluidics aerator in operation (supplied by Pfleiderer water systems $\mathrm{GmbH}$, Germany). Air passes through pores of equivalent diameter $120 \mu \mathrm{m}$, between sintered polyethylene spheres. Bubbles are generated at only a few exits - due to the phenomenon called instability of parallel bubble formation (Tesař 2007) — and are very large: the Sauter mean $5.7 \mathrm{~mm}$ bubble diameter evaluated in this picture is 47-times larger than the equivalent diameter of pore exits

\section{HIGH-SPEED-CAMERA IMAGE STUDIES}

Because of the absence of reliable information about generation of microbubbles, author (Tesař, 2013a) begun series of experimental studies in an attempt to understand the mechanism. All previous researchers so far were satisfied in their studies of bubble (and microbubble) formation with visual observations or images obtained using a standard-speed camera. However, the repetition rates of microbubble formation are often very much higher of the order $10^{2} \mathrm{~Hz}$ - than what can be visually captures. Author was able to use for his study of microbubble formation a highspeed camera, capable of taking images at 4000 frames per second or more - the limiting factor being the available light that had to be focused onto the very small millimetre-sized picture scene. The key component of the image taking was a longdistance macro-lens Machine Vision "Navitar" 12X. This made possible photographs of sub-millimetre objects from a distance as large as $100 \mathrm{~mm}$ from the front lens. This allowed taking the highspeed video sequences from outside of the water tank, through its glass walls. Also, this special lens made possible clear recognition of details at extremely small distances from the exits of the aerator passages. It was in particular the phenomena observed in this region that provided an explanation to the apparent paradox of the microbubble diameters much larger than the equivalent diameter 
of the aerator passage exit (Tesař, 2013a).

The explanation was found in conjunctions of freshly formed microbubbles - merging a certain number of microbubbles to form a single much larger bubble. The key factor is the fact that ascent velocity of bubbles decreases rapidly with decreasing size. Microbubbles move so slow they do not get far from the passage exit before the next, follower microbubble appears, gets into touch with the already present one, and merge with it. The merging is favoured from the energy point of view - some energy is released as is seen from the following consideration of a spherical bubble of diameter $d_{j}$ has volume:

$V=\frac{\pi}{6} d_{j}^{3}$

and its surface area is:

$F=\pi d_{j}^{2}$

so that its surface energy is:

$E=\pi d_{j}^{2} \sigma$

where $\sigma\left[\mathrm{N} / \mathrm{m}^{2}\right]$ is the surface tension.

Conjunction of two equal-sized bubbles of diameter $d_{j}$ thus results in a single bubble of diameter $d c$ and volume:

$V_{c}=\frac{\pi d_{j}^{3}}{3}=\frac{\pi d_{c}^{3}}{6}$

and hence surface area:

$F_{c}=\pi d_{c}^{2}=2^{\frac{2}{3}} \pi d_{j}^{2}$

while the total surface of the two small pre-conjunction primary microbubbles was:

$F_{\text {total }}=2 \pi d_{j}^{2}$

This was $2^{\left(-\frac{1}{3}\right)}=0.794-$ times larger. Thus, there is energy:

$\Delta E=\left(2-2^{\frac{2}{3}}\right) \pi d_{j}^{2} \sigma$

released during this conjunction. It is not small - its magnitude is more than one fifth of the available total sum of surface energy of the two pre-conjunction microbubbles. The energy eq. (7) is dissipated by viscous effects during the strong oscillation that takes place immediately after the conjunction. Analysis of highspeed camera images has shown, as documented in the example presented in Fig. 2, that the after-conjunction oscillating motion effectively stops the upwards motion of the larger bubble formed in the process. This larger bubble thus stays for some time practically at the same height above the aerator top surface - and, as a result, inevitably takes place in another conjunction with the subsequently formed next bubble. In the example presented in Fig. 2 there were five microbubbles taking part in the four conjunctions $A, B, C$, and $D$ before the resultant bubble was finally released and moved away. In other observed cases the number of repeated conjunctions was even much higher. Note in Fig. 2 that all this happened at very small distances - merely around 0.2 $\mathrm{mm}$ - above the aerator exit and the conjunctions followed one another at $\sim 6$ millisecond intervals. Both the position and speed made it impossible to watch the processes visually. No wonder the phenomenon has so far escaped attention of earlier researchers.

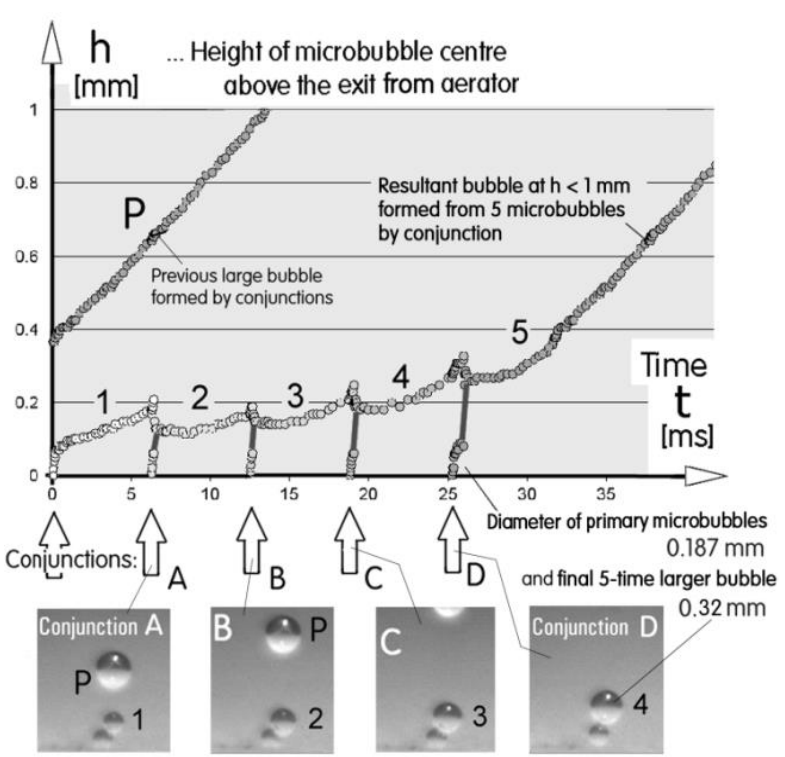

Fig. 2. An example of high-speed camera images (bottom) of freshly produced microbubbles $1,2,3,4$, and 5 - and a diagram of evaluated heights $h$ above the aerator exit as a function of time $t$. Microbubbles move upwards so slowly that they get into contact and subsequent conjunction with the next, later generated microbubble. The conjunctions may be repeated several times before the microbubble is finally released

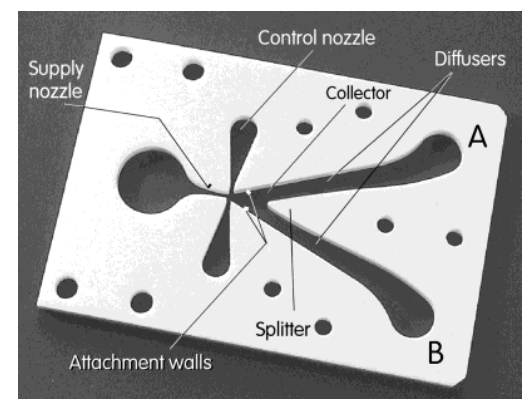

Fig. 3. A typical example of a fluidic amplifier (Tesař, 2009) of the jet-deflection type. When provided with suitable de-stabilising feedback loop channels it may be the essential part of a fluidic oscillator

\section{STANDARD FLUIDIC OSCILLATORS}

\subsection{Amplifier and feedback loop}

According to Zimmerman et al., 2008, the bubble growth caused by the conjunctions may be eliminated if the gas flow is pulsed, preferably by a fluidic no-moving-part oscillator. The problem that remained was the uncertainty: sometimes the pulsation did help, sometimes not. The reason is in the character of the action of the oscillator.

The self-excited fluidic oscillators in general use the hydrodynamic instabilities in the flowfield. There is a large number of alternative possibilities how to create the instabilities. Most often used are version consisting (Tesař, 2013b) of two components. The first component is a fluidic amplifier - a device responding by large changes of the conditions in its output terminal to a rather weak control fluid flow into its input. The second component are destabilising negative feedback loops connecting the amplifier inputs and outputs, Fig. 4. 

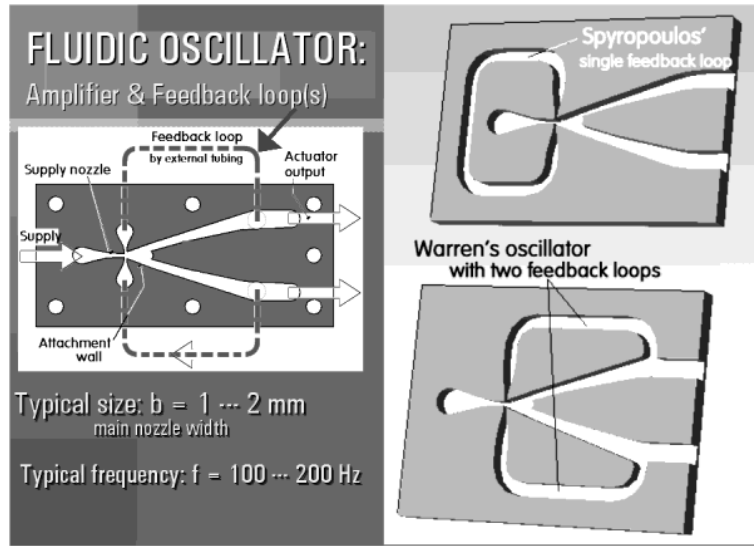

Fig. 4. Usual versions of fluidic oscillators with two alternative layouts of feedback loops, both invented in the 1960's by Warren. These oscillators sometimes did produce microbubbles - but sometimes did not. To discover the reasons, author investigated the mechanism of bubble formation using a high-speed camera

An example of typical amplifier is presented in Fig. 3. It shows the main plate in which are made cavities for air flows. In operation, the cavities are closed by being covered by flat top and bottom cover plates. The amplifier shown is of the jet-deflection Coanda-effect bistable type. At left in Fig. 3 is supply nozzle into which is fed a constant flow of air. This flow leaves the supply nozzle as a jet issuing into the cavity between two mutually opposed attachment walls. The apex angle of these two walls is so large that the jet cannot follow both. Instead, by a phenomenon known as the Coanda effect, the jet attaches to only one attachment wall - and is led through one of the two diffusers into either A or B output terminal, seen at the right-hand side of the picture. On each side of the supply nozzle exit in Fig. 3 there are control nozzles. Air flow admitted into one of these nozzles - the one at the side to which the jet is attached - causes the main jet (the one leaving the supply nozzle) to switch from the ON one attachment wall to the other, opposite one that was so far in the OFF state.

Converting a fluidic amplifier into an oscillator is achieved by the feedback loops. Two most popular loop versions are presented in Fig. 4. Both lead to a de-stabilising effect: if the flow passes through the amplifier in Fig. 3 to the terminal $A$, then a signal is fed into the control nozzle on the same. This switches after a certain time delay - the main jet flow into the other terminal $B$. The roles of the terminals are then exchanged and this is a beginning of another oscillation period.

It should be noted that the Coanda-effect attachment of the main jet to the attachment wall requires a low resistance load connected to the amplifier output terminals. If the flow through e.g. the terminal A encounters a high-resistance loading (e.g., is to pass through a connected device with small channel cross sections), the Coanda effect fails to keep the main jet deflected. If there is no such load connected to the other output terminal, the jet switches to that easier flow path - and becomes attached to the other attachment wall. The load-switched fluidic amplifiers therefore need no control nozzles.

\subsection{Flow reversal caused by jet pumping}

It is useful to note that the main jet (as all jets do, especially if their Reynolds number is high enough so that the flow is turbulent) entrains the air from its surrounding space and carries it away. Let us assume that the jet leaves the amplifier through the output terminal $A$ in Fig. 3. Its entrainment action removes the surrounding fluid from the amplifier - where it is replaced by a suction inflow into the amplifier body through the other terminal $B$. This suction effect of the ON jet flow acting in the other, OFF terminal is called jet-pumping.

If the amplifier is provided with the feedback and oscillates, this jet-pumping effect generates in its output terminals an alternation of suction and blowing, each for a part of oscillation period. This temporary flow reversal, however, requires low-resistance loads connected to the terminals. The lack of success some microbubble researchers encountered with their fluidic oscillators was due to the high resistance of their aerators (too small and too long passages). What took place instead of the jet pumping was spillover flow of periodically varied intensity through the OFF terminal. The adjustment of the oscillator and load for their proper collaboration requires a matching similar to the one discussed in Tesař (2009).

\section{SUPPRESSING THE CONJUNCTION BY THE OSCILLATOR}

\subsection{High-speed camera images of successful cases}

For the studies of processes at (as well as in) the aerator passages was designed a laboratory model aerator with well ordered passages, made by drilling (rather than the chaotically arranged passages usually made by sintering of small particles, as is the example case shown in Fig. 1). The organised aerator layout is, of course, amenable to better description and study. In the model aerator there was a set of parallel drilled holes. To avoid expected problems with breaking the very small drill bits, the holes were of relatively large, $0.6 \mathrm{~mm}$ diameter - Fig. 5 . Also, to make the process of microbubble generation easily adjustable, the fluidic oscillator was for this study replaced by a pulsation generator with a generator of harmonic electric signal and a loudspeaker.

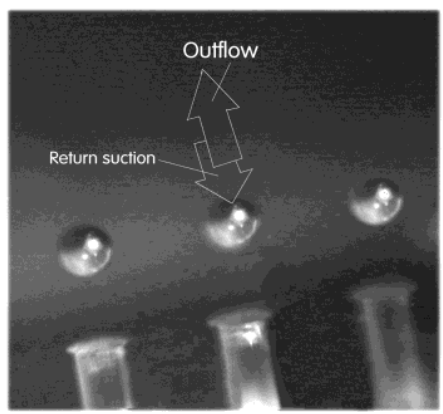

Fig. 5. An example of high-speed camera images of freshly produced microbubbles that just left the row of $0.6 \mathrm{~mm}$ diameter aerator passages. The conjunctions shown in Fig. 2 were in this case eliminated because the used oscillator caused flow direction reversal for a part of the cycle. Thus the newly formed microbubbles having returned into their aerator passage are protected from contact with the microbubble previously made

Successful generation of microbubbles with this model is seen in the microphotograph in Fig. 5. The amplitude of the oscillation was so large that the microbubble motion was not at all dependent upon the small steady ascent velocity. Instead, the microbubble motion was governed by a mechanism similar to the processes 
in a synthetic jet (Trávníček et al. 2007, Tesař 2007): entrainment of fluid near to the exit and a rectified one-direction flow at larger distances. The freshly produced microbubble after its emergence from the aerator is in the subsequent part of the oscillatory period sucked back into the aerator passage - while its predecessor microbubble at a larger axial distance from the passage exit is not influenced by the suction a and moves away. The motion is governed by the inertia of water also moved (this water gets into the aerator passage during the previous suction part of the cycle from locations near to the passage exit.

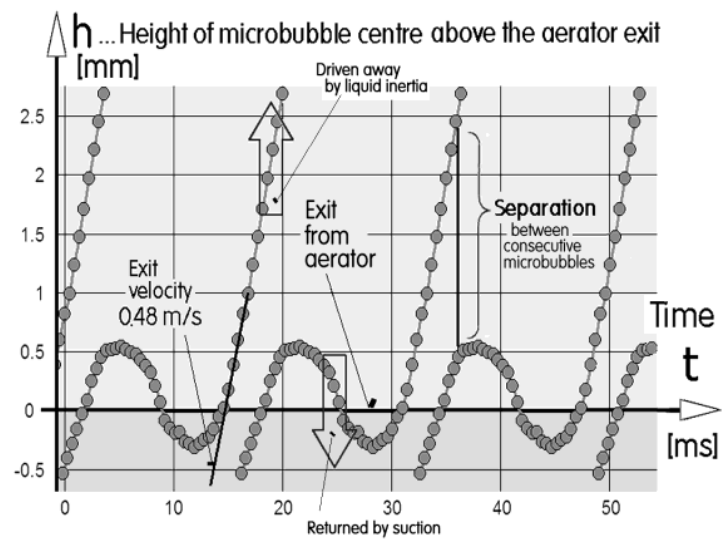

Fig. 6. Height vs. time trajectories of freshly formed microbubbles seen in Fig. 5 - i.e. those influenced by the temporary flow reversals caused by the jet-pumping in the fluidic oscillator

The model generated small microbubbles of the diameter $0.6 \mathrm{~mm}$, the same as that of the passage exits - and very much smaller than the typical bubbles obtained with contemporary steady-flow aerators (Fig. 1). The generation of microbubbles was also studied by analysis of high-speed camera images (analogous to those described above in Section 2 for the steady, nonpulsating air flows). With the applied pulsation, there were no observable microbubble conjunctions. It was soon found that this absence of conjunctions (which keeps the bubbles small) is due to the temporarily reversing character of the flow direction in the aerator passages. The reversal is seen in the time history diagram presented in Fig. 6. The pulsation amplitude is seen to be so large that during a part of the oscillation cycle the suction moving the microbubble back into the aerator passage prevents it from getting in touch with the previously generated ones. Also of importance is the suction of the water from the vessel into the passage - as is shown e.g. in the regime $C$ in Fig. 7 . In the following part of the oscillation cycle this water column from the passage is expulsed, together with the microbubble. It is essential to note that the density of the bubble is roughly three orders of magnitude smaller than density of water. It would be therefore wrong to think about acceleration of the bubble. Instead, it is the water column inside the aerator passage that is accelerated (in the regime $D$ in Fig. 7). Its inertia moves it together with the microbubble quite far from the aerator. This way is suppressed the effect of the small ascent velocity and consequent mutual contact that without oscillation leads to the conjunctions.

It should be noted that the diagram in Fig. 6 shows the vertical position of the bubble centre as a function of time. The lines connecting in Fig. 6 the locations of the microbubble centres may suggest that the microbubbles having left the passages are not particularly much separated in space in the horizontal direction. This would be a wrong impression, on the horizontal co-ordinate is time. What really influences the possibility of the mutual conjunctions are the vertical separations of the bubbles - and these separations are large.

\subsection{Failures caused by improper loading the oscillator}

With this demonstrated elimination of conjunctions - and the clear explanation of the elimination phenomenon - it became a question why the positive results were obtained only in some tests. The reason was found in disappearance of the jet-pumping phenomenon if the oscillator is loaded by high hydraulic resistance.
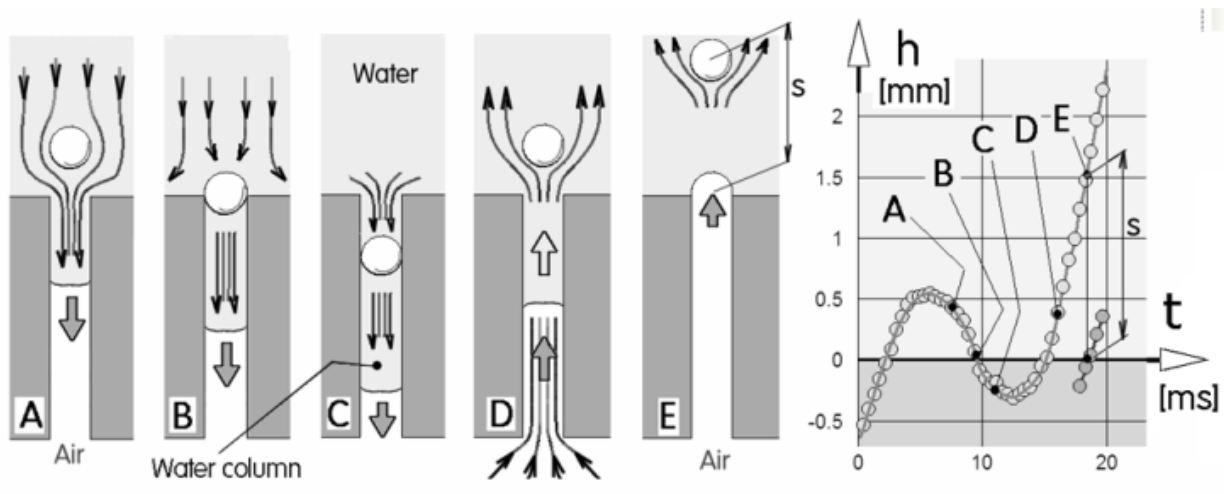

Fig. 7. The states from $A$ to $E$ eat left show schematically the water pathlines and positions of a microbubble and water column during the oscillation cycle - the instants are identified in the diagram from Fig. 6.

In the successful tests discussed in Section 4.1 the jet pumping did occur due to two factors. First, the used oscillator was quite small in relation to the rather large aerator's drilled passages. The aerator thus represented a low-resistance load. Second, the hydrostatic pressure acting on the submerged aerator passage exits did not add much to the loading because - for easier observation - the aerator was placed to only a small depth under the water surface. These two favourable factors disappeared in the unsuccessful tests with larger amplifiers ( the size chosen for generating more microbubbles) and aerators with relatively long, tortuous passages positioned in the usual large depths. 


\section{ELIMINATING THE CONJUNCTIONS}

\subsection{Fluidic circuit with jet pump and accumulator}

The solution that made the reverse flow for a part of oscillation cycle reliably present was provided with the fluidic oscillator designed specifically for generation of micro-bubbles. It is a subject of author's recent patent application (Tesař, 2015). The flow reversal in this device does not depend on the rather weak, generally unreliable and sometimes non-reproducible effect of jet pumping inside the amplifier. Instead, the return flow is driven by energy of air accumulated inside a fluidic accumulator, which is a part of the circuit. Also, this available driving effect is employed in an efficient, full-scale jet pumping.

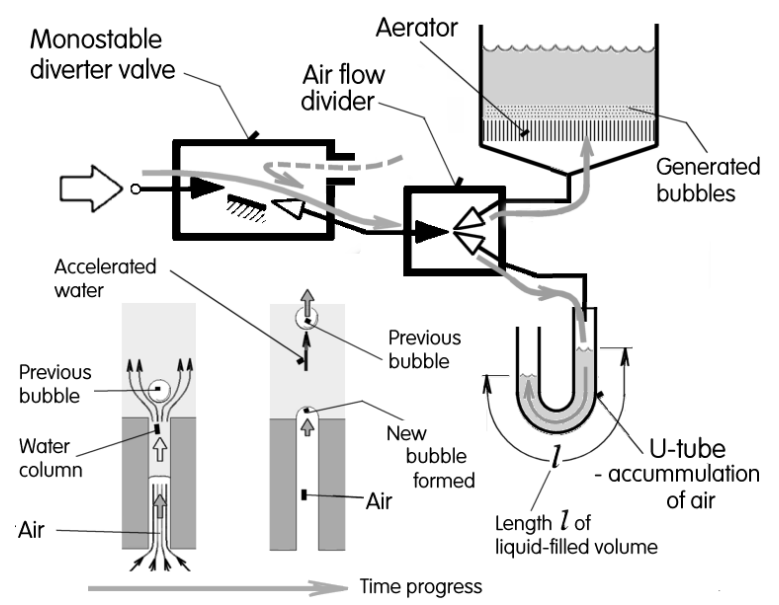

Fig. 8. Schematic representation of the fluidic generator according to the author's patent application (Tesař, 2015). The two details drawings in the bottom left are the cases $D$ and $E$ from Fig. 7 . They show the character of the flow in an aerator passage in the half-period characterised by generation of the microbubbles

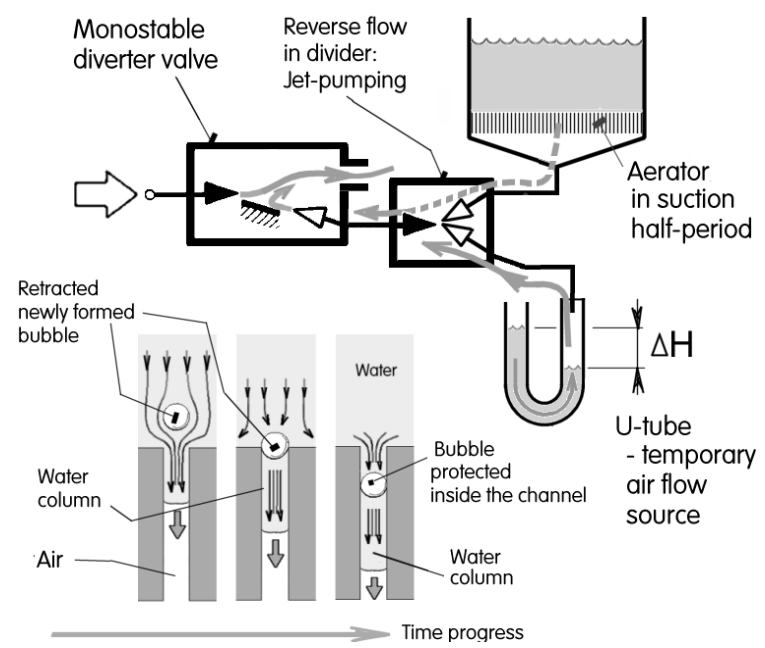

Fig. 9. Schematic representation of the same generator as in previous Fig. 8 - here, however, showing the half-period of air flow back from the water tank into the aerator passages

In one of its simplest versions the new layout is presented schematically in Figs. 8 and 9. The air flows are there indicated gray arrows. The components of the schematically presented devices are nozzles, drawn as black (fully filled) triangles, - and diffusers, drawn schematically as white (not filled) triangles. The first picture, Fig. 8, shows the arrows in the expulsion half-period, i.e. in the part of the cycle during which air is percolated through the aerator passages into the water in a large vessel, with the aerator at its bottom. The other Fig. 9 shows the air flow arrows in the other, suction half-period. In both pictures in their left-hand lower part are added the corresponding representations of the flow in the aerator passages from Fig. 7.

The air supplied into the microbubble generator comes first into a device drawn in the upper left corner of the circuit. It is a loadswitched monostable diverter. In principle it may be described as akin to jet-deflection type fluidic diverter amplifiers discussed in Section 3 - the difference being in there being only one attachment wall and also in the absence of control nozzles. This device is switched by the load-switching effect discussed above. The monostability means that the air jet generated in the supply nozzle tends to attach always to the single attachment wall. There is also only one output terminal - apart from it, there is in this device a vent outlet, connecting the internal cavities of the device with the atmosphere outside.

The other part is the jet pump device, located in the centre of the picture. It possesses one nozzle and two diffusers, with their smaller cross section ends at the nozzle. The nozzle is connected to the output of the diverter device. One output terminal of the jet pump (i.e. the larger cross-section end of the diffuser) is connected to the aerator at the bottom of the water-filled vessel. The other output terminal is connected to the fluidic accumulator. There are many alternative designs of fluidic accumulators. The one chosen for schematic presentation in Figs. 8 and 9 is the U-tube with two vertical columns - one of them open into atmosphere.

In the situation presented in Fig. 8, air jet issuing from the supply nozzle at left attaches to the attachment wall and is guided into the nozzle of the jet pump - which in this regime does not operate as a jet pump at all - its role may be described as divider, dividing the supplied ait flow into two output flows. The dashed arrow line passing through the vent outlet of the diverter indicates that there may be some jet pumping suction effect. It is an effect, however, which is here unimportant; the discussed fluidic circuit is capable of performing its task even if the pressure inside the device is higher than atmospheric and some air actually leaves the cavity through the vent as a spillover flow.

One of the output flows leaving the exits of the jet-pump /f lowdivider progresses to the aerator where it generates the small bubbles. The flow from the other exit continues into the active arm of the U-tube, where it gradually pushes down the water level. This, of course, is associated with rising water level in the other arm. Thus the pressure in the active arm gradually increases and finally reaches the level at which the load-switching occurs in the monostable diverter valve.

After the switching, the air jet in the diverter separates from the attachment wall - as shown in Fig. 9 - and leaves through the vent into atmosphere. This is the beginning of the other halfperiod. Between the both arms of the U-tube now exists the pressure difference:

$\Delta P=g \Delta H$,

where $\mathrm{g}=9.81 \mathrm{~m} / \mathrm{s}^{2}$ is the gravitational acceleration. This difference drives the air away from the U-tube into the jet pump/ divider which now behaves as the usual jet pump, i.e. entrains the air 
from the aerator and takes it along to the switched divider. . There this return flow prevents the main air jet from attaching to the attachment wall (i.e. keeps the air flow deflected into the vent). This is the desirable regime with removal of the freshly made microbubbles back into the aerator passages. Of course, as the time progresses, the height $\Delta H$ (as well as $\Delta P$, eq. (8) ) in the $\mathrm{U}$ tube decreases. Finally the influence of this flow from the $U$-tube accumulator ceases completely. There is no more the reverse flow that could maintain the main air jet in the monostable diverter separated from the attachment wall. The jet clings back to the wall — and the next oscillation cycle may begin.

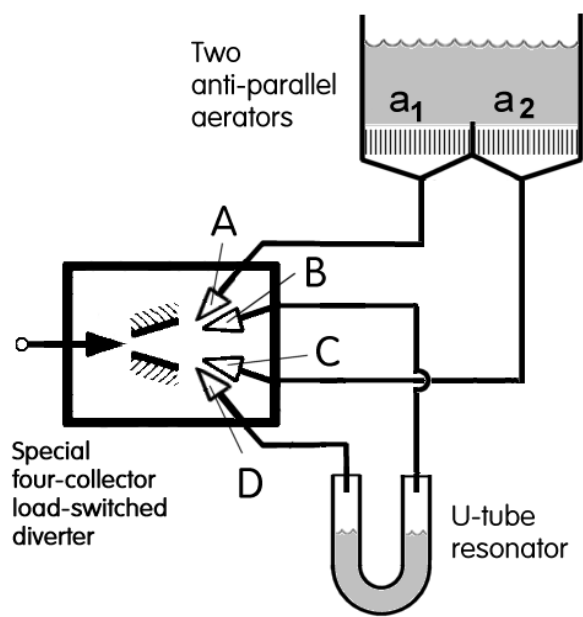

Fig. 10. Schematic representation of the double-acting fluidic generator of microbubbles with prevention of the conjunctions. The diffusers $A$ to $D$ are the ones presented in Fig. 11

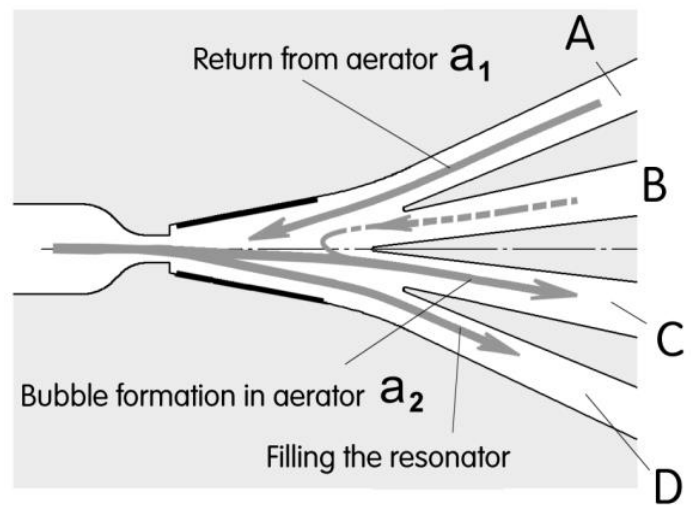

Fig. 11. Interaction cavity of the special diverter with four collectors $A$ to $D$. Grey arrows indicate the character of air flow in the half-period of formation of microbubbles in the aerator $\mathrm{a}_{2}$

The periodic switching between the two regimes is in tune with the oscillatory motions of the liquid in the U-tube - the frequency of which is:

$f=\frac{1}{2 \pi} \sqrt{\frac{g}{l}}$,

where $l$ is the length of the liquid column in the U-tube, as shown in Figs. 9, 14.

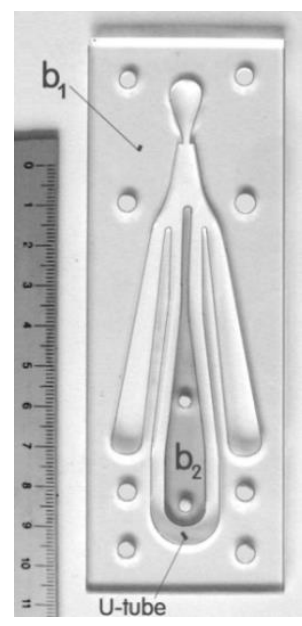

Fig. 12. Photograph of the two components ( $b_{1}$ and separate inner component $b_{2}$ ) of the laboratory model of the double-acting microbubble divider - which now really operated as the jet pump. From there it generator with integral U-tube resonator. The air and water flow cavities were laser-cut in $3 \mathrm{~mm}$ thick polymethylmethacrylate plate

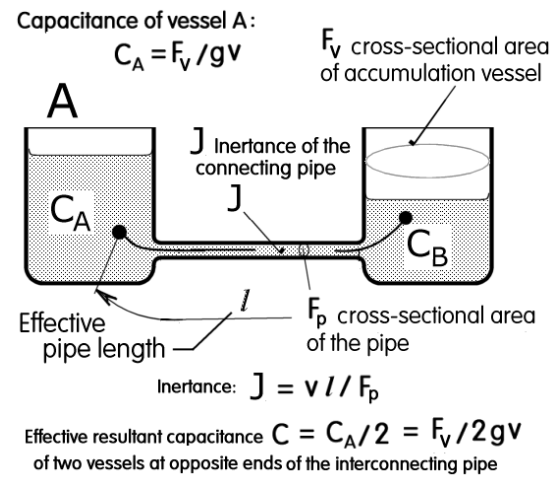

Fig. 13. The principle of the CJC oscillator: two accumulation cavities (of capacitance values CA and CB respectively) are connected by a pipe of inertance $J$

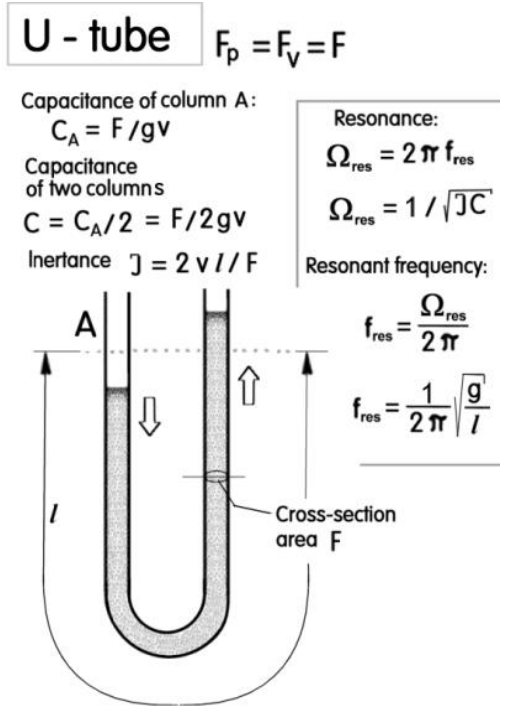

Fig. 14. The U-tube oscillator: contrary to the separate accumulation and inertia components in Fig. 14, here the two vertical columns share the capacitance as well as inertance properties 


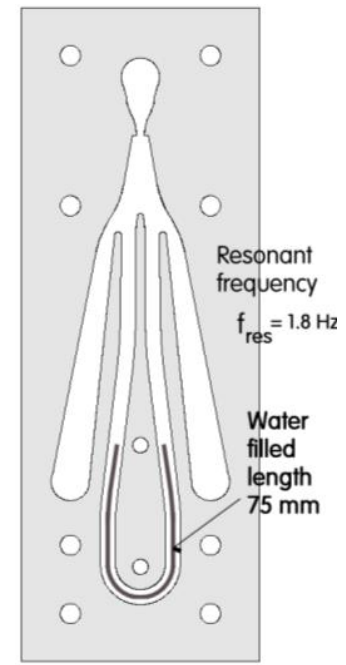

Fig. 15. Early tests with the U-tube integrated into a load-switched fluidic jet-type oscillator verified the capability of self-excited oscillation at very low frequencies

\subsection{Integral design}

Obvious disadvantage of the circuit discussed in the previous Sect 5.1 is the energetic loss associated with allowing the compressed air to leave into the atmosphere (through the vent of the divider) during approximately one half of the oscillation cycle. This is actually not necessary. The monostable layout was chosen for discussion here only because of its simplicity and ease of explanation of its action. There may be a double-acting configuration with a single U-tube resonator - but with two aerators, operating in anti-parallel (i.e. in opposite phases).

Another source of losses that may be eliminated is the connection between the monostable diverter valve and the jet pump device. The air flow is first slowed down in the diffuser of the monostable device - only to be accelerated in the nozzle of the jet pump a short distance downstream. Of course, these two conversions are inevitably associated with hydraulic losses.

Figs. 10, 11, 12, and 13 present an example of layout also discussed in Tesař (2015) that removes both above mentioned shortcomings. Of course, while the devices used in the circuit presented in Figs. 8 and 9 may be standard fluidic devices, the multi-collector integral design in Figs. 10 and 11 are unusual, specially designed for the purpose. The development of the oscillator thus may be perhaps more difficult.

\section{CONCLUSIONS}

Generation of microbubbles has very large potential importance for future process engineering. Especially, the potential importance of producing renewable automobile fuels may be very high. Particularly attractive - inexpensive and reliable - are microbubble generation methods based on pulsating the gas flow by fluidic oscillators. Until recently, however, this generation method was not always successful, the generated bubbles being large. High-speed camera studies pinpointed the reason: it was the consequence of multiple conjunction of microbubbles immediately after their formation. Also found was the solution of the problem: reversing the flow direction for a part of the oscillation period. An oscillator design is described producing this desirable effect by a U-tube resonator integral within the oscillator body. It is a subject of a recent patent application as well as tests in laboratory.

\section{REFERENCES}

1. Allouch A., Bourmine K., Monmayrant A., Gauthier-Lafaye o., Geoffroy S., Guo A.-M., Joseph P. (2014), Microbubbles for optofluidics: controlled defects in bubble crystals, Microfluidics and Nanofluidics, 549-560.

2. Al-Mashhadani M.K.H., Wilkinson S.J., Zimmerman W.B. (2015), Airlift bioreactor for biological applications with microbubble mediated transport processes, Chemical Engineering Science, Vol. 137, 243253.

3. Al-Mashhadani M.K.H., Bandulasena H.C.H., Zimmerman W.B. (2012), CO2 mass transfer induced through an airlift loop by a microbubble cloud generated by fluidic oscillation, Industrial and Engineering Chemistry Research, Vol. 51, 1864-1877.

4. Bogdevich V.G., Evseev A.R.., Mljuga A.G., Migirenko G. S. (1978), Gas saturation effect on near-wall turbulence characteristics, Proc of 2nd International Conference on Drag Reduction, Cambridge, BHRA, 25-34.

5. Coward T., Lee J. G. M., Caldwell G.S. (2015), The effect of bubble size on the efficiency and economics of harvesting microalgae by foam flotation, Journal of Applied Phycology, Vol. 27, 733-742.

6. Demirbas A., Demirbas M.F. (2011) Importance of algae oil as a source of biodiesel, Energy Conversion and Management, Vol. 52, 163-170.

7. Hanotu J., Bandulasena H.C.H., Zimmerman W.B. (2012), Microflotation performance for algal separation, Biotechnology and Bioengineering, Vol. 109, 1663-1673.

8. Hanotu J., Bandulasena H.C.H., Chiu T. Y., Zimmerman W.B. $\mathbf{t}(2013)$, Oil emulsion separation with fluidic oscillator generated microbubbles, International Journal of Multiphase Flow, Vol. 56, 1191125.

9. Hashimoto M., Mayers B., Garstecki P., Whitesides G. M. (2006), Flowing lattices of bubbles as tunable, self-assembled diffracting gratings, Small, Vol. 2, 1292-1298

10. Hu X., Liu B., Zhou J., Jin R. Qiao S., Liu G. (2015), $\mathrm{CO}_{2}$ fixation, lipid production, and power generation by a novel air-lift-type microbial carbon capture cell system, Environmental Science and Technology, Vol. 49, 10710-10717.

11. James A., Vukasinovic B., Smith M. K.,Glezer A, (2003), Vibration-induced drop atomization and bursting, Journal of Fluid Mechanics, Vol. 476, 1-28.

12. Jones S.M.J., Harrison S.T.L. (2014), Aeration energy requirements for lipid production by Scenedesmus sp. in airlift bioreactors, Algal Research, Vol. 5, 249-257.

13. Kanagawa T. (2013), Focused ultrasound propagation in water containing many therapeutical microbubbles, Paper OS6-04-4, Proc. of FLUCOME 2013, 12th Intern. Conf., Nara, Japan

14. Kargbo D.M. (2010), Biodiesel production from municipal sewage sludges, Energy and Fuels, Vol. 24, 2791-2797.

15. Kooiman K., Foppen-Harteveld M., Der Steen A.F.W.V., De Jong N.(2011), Sonoporation of endothelial cells by vibrating targeted microbubbles, Journal of Controlled Release, Vol. 154, 35-41..

16. Kuznetsova L.A., Coakley W.T. (2007), Applications of ultrasound streaming and radiation force in biosensors, Biosensors and Bioelectronics, Vol. 22, 1567-1572.

17. Lam M.K., Lee K.T, (2012) Microalgae biofuels: a critical review of issues, problems and the way forward, Biotechnology Advances, Vol. 30, 673-678.

18. Lee J.H., Lee K. H., Won J. M., Rhee K., Chung S. K. (2012), Mobile oscillating bubble actuated by AC-electrowetting-on-dielectric for microfluidic mixing enhancement, Sensors and Actuators A: Physical, Vol. 182, 153-162. 
19. Leite G.B., Abdelaziz A.E., Hallenbeck P.C. (2013), Algal biofuels: challenges and opportunities, Bioresource Technology, Vol. 145, 134-139.

20. Madavan N.K., Deutsch S., Merkle C. L. (1984), Reduction of turbulent skin friction by microbubbles, Physics of Fluids, Vol.27, 356-363.

21. McCormick M.E, Bhattacharyya R. (1973), Drag reduction of a submersible hull by electrolysis, Naval Engineers Journal, Vol. 85 , 2973-2978

22. Moriguchi Y., Kato H. (2002), Influence of microbubble diameter and distribution on frictional resistance reduction, Journal of Marine Science and Technology, Vol. 7, 79-85.

23. Oh J.S., Kwon Y. S., Lee K. H., Jeong W., Chung S. K., Rhee K. (2014), Drug perfusion enhancement in tissue model by steady streaming induced by oscillating micro-bubbles, Computers in Biology and Medicine, Vol. 44, 37-43

24. Pang M.J., Wei J.J., Yu B. (2014), Numerical study on modulation of microbubbles on turbulence frictional drag in a horizontal channel, Ocean Engineering, Vol. 81, 58-64.

25. Prevenslik T. (2011), Stability of nanobubbles by quantum mechanics, Proceedings of conference 'Topical Problem of Fluid Mechanics', Prague, 113-116.

26. Rawat I., Ranjith Kumar R., Mutanda T., Bux F. (2011) Dual role of microalgae: phycoremediation of domestic wastewater and biomass production for sustainable biofuels production, Applied Energy, Vol. 88, 3411-3424.

27. Rehman F., Medley G. J. D., Bandulasena H.C.H., Zimmerman W. B. (2015) Fluidic oscillator-mediated microbubble generation to provide cost effective mass transfer and mixing efficiency to the wastewater treatment plants, Environmental Research, Vol.137, 3239.

28. Rodríguez-Rodríguez J., Sevilla A., Martinez-Bazán C., Gordillo J. M. (2015), Generation of microbubbles with applications to industry and medicine, Annular Review of Fluid Mechanics, 405-429.

29. Shams M.M., Dong M., Mahinpey N. (2014), Friction factor of microbubbles in capillary tubes at low Reynolds numbers, Chemical Engineering Science, Vol.112, 72-77.

30. Sun R.R., Noble M. L., Sun S. S., Song S., Miao C. H. (2014), Development of therapeutic microbubbles for enhancing ultrasoundmediated gene delivery, Journal of Controlled Release, Vol. 182 $111-120$

31. Terasaka K., Hirabayashi A., Nishino T., Fujioka S., Kobayashi D. (2011), Development of microbubble aerator for waste water treatment using aerobic activated sludge, Chemical Engineering Science, Vol. 66, 3172-3179.

32. Tesař V., Tippetts J. R., Allen R. W. K., Low Y.-Y. (2005), Subdynamic asymptotic behavior of microfluidic valves, Journal of Microelectromechanical Systems, Vol. 14, 335-347.

33. Tesař V. (2007), Configurations of fluidic actuators for generating hybrid-synthetic jet, Sensors and Actuators A: Physical, Vol. 138, 394-403.

34. Tesař V. (2007), Fluidics applied to generating small aeration bubbles, Proc. of 9th Int. Symp. FLUCOME 2007, Tallahassee, FLA USA.

35. Tesař V. (2009) Fluidic control of reactor flow - Pressure drop matching, Chemical Engineering Research and Design, Vol. 87, 817832.

36. Tesař V. (2009), Enhancing impinging heat or mass transgfer by fluidically generated flow pulsation, Chemical Engineering Research and Design, Vol. 87, 181-192.

37. Tesař V. (2010), No-moving-part valve for automatic flow switching, Chemical Engineering Journal, Vol. 162, 278-295.

38. Tesař V. (2013), Microbubble smallness limited by conjunctions, Chemical Engineering Journal, Vol. 231, 526-536.

39. Tesař V. (2014), Microbubble generator excited by fluidic oscillators's third harmonic frequency, Chemical Engineering Research and Design, Vol. 92, 1603-1615.
40. Tesař V. (2014a) New concept: Low-pressure wide-angle atomiser, Chemical Engineering and Processing: Process Intensification, Vol. 82, 19-29.

41. Tesař V. (2014b), Shape oscillation of microbubbles, Chemical Engineering Journal, Vol. 235, 368-378.

42. Tesař V. (2015), Fluidic generator of microbubbles (in Czech), Czech Rep. Patent Application, PV 2015-204 filed March 2015.

43. Tesař V., Hung C.-H., Zimmerman W.B.J. (2006), No-moving-part hybrid-synthetic jet actuator, Sensors and Actuators, A: Physical, Vol. 125, 159-169.

44. Tesar V., Zhong S. (2003), Efficiency of Synthetic Jet Generation, Transactions of the Aeronautical and Astronautical Society of the Republic of China, Zhongguo Hangkong Taikong Xuehui Huikan, Vol. 35, 45-53.

45. Tesař V., Zhong S., Fayaz R. (2013) New fluidic oscillator concept for flow separation control, AIAA Journal, Vol. 51, 397-405

46. Trávníček Z., Tesař V., Kordk J. (2007), Performance of synthetic jet actuators based on hybrid and double-acting principles, Journal of Visualization, Vol.11, 221-I220.

47. Tremblay-Darveau C., Williams R., Burns P.N. (2014), Measuring absolute blood pressure using microbubbles, Ultrasound in Medicine and Biology, Vol. 40, 775-781.

48. Tsuge H., Li P., Shimatani N., Shimamura Y., Nakata H., Ohira M. (2009) Fundamental study on disinfection effect of microbubbles, Kagaku Kogaku Ronbunshu, Vol. 35, 548-552.

49. Wang C., Yalikop S. V., Hilgenfeldt S. (2012), Efficient manipulation of microparticles in bubble streaming flows, Biomicrofluidics, Vol. 6, 012801

50. Watanabe O., Masuko A., Shirose Y. (1998), Measurements of drag reduction by microbubbles using very long ship models, Journal of Soc. Naval Architects, Vol. 183, 53-59.

51. Watanabe Y., Aoi A., Horie S., Tomita N., Mori S., Morikawa H., Matsumura Y., Vassaux G., Kodama T. (2008), Low-intensity ultrasound and microbubbles enhance the antitumor effect of cisplatin, Cancer Science, Vol. 99, 2525-2531.

52. Wataneabe K., (2013), Washing effect of microbubbles, Paper OS101-1, Proc. of FLUCOME 2013, 12th Intern. Conf., Nara, Japan, November 2013

53. Xi X. (2012), Controlled translation and oscillation of microbubbles near a surface in an acoustic standing wave field, PhD Thesis, Mechanical Engineering Department, Imperial College London.

54. Yanuar, Gunawan, Sunaryo, Jamaluddin A. (2012), Micro-bubble drag reduction on a high-speed vessel model, Journal of Marine Science and Technology, Vol. 17, 301-304

55. Zimmerman W.B., Tesař V., Butler S., Bandulasena H.C.H. (2008), Microbubble generation, Recent Patents in Engineering, Vol. 2, 1-8

56. Zimmerman W.B., Al-Mashhadani M.K.H., Bandulasena H.C.H. (2013) Evaporation dynamics of microbubbles, Chemical Engineering Science, Vol. 101, 865-877.

57. Zimmerman W.B., Zandi M., Bandulasena H.C.H. (2011), Towards energy efficient nanobubble generation with fluidic oscillation, Current Opinion in Colloid \& Interface Science, Vol. 16, 350-356.

58. Zimmerman W.B., Zandi M., Bandulasena H.C.H., Tesa5 V., Gilmour J.D., Ying K. (2011), Design of an airlift bioreactor and pilot scale studies with fluidic oscillator induced micro bubbles for growth of a microalgae Dunaliella Salina, Applied Energy, Vol. 88, 3357-3369.

Acknowledgements: Author gratefully acknowledges the support by grant Nr. 13-23046S obtained from the GACR - Grant agency of the Czech Republic, and the institutional support RVO: 61388998. 